H. E. Scholtz • S. G. Pretorius • D. H. Wessels •

R. H. A. Becker

\title{
Pharmacokinetic and glucodynamic variability: assessment of insulin glargine, NPH insulin and insulin ultralente in healthy volunteers using a euglycaemic clamp technique
}

Received: 15 December 2004 / Accepted: 16 May 2005 / Published online: 14 September 2005

C) Springer-Verlag 2005

\begin{abstract}
Aims/hypothesis: This single-dose, doubleblind, randomised, parallel-group study evaluated the reproducibility in systemic exposure and glucodynamic effect of insulin glargine, NPH insulin (NPH) and insulin ultralente (ultralente) using the manually adjusted euglycaemic clamp technique. Methods: In total, 36 healthy volunteers received two consecutive s.c. injections $(0.4$ $\mathrm{IU} / \mathrm{kg}$ ) of glargine, NPH or ultralente with a wash-out period of 7 days between treatments. Results: In healthy volunteers, glargine presented well-reproduced flat concentration profiles and no pronounced peaks in activity. NPH, by contrast, showed well-defined peaks in concentration and glucose disposal, while ultralente had highly variable profiles. Within-subject variability (ANOVA) for insulin exposure over $24 \mathrm{~h}$ was $15 \%$ for glargine and $19 \%$ for $\mathrm{NPH}$, compared with $67 \%$ for ultralente $(p<0.05$, glargine and NPH vs ultralente). The $49 \%$ within-subject variability in total glucose disposal (glucose infusion rate [GIR]-AUC ${ }_{0-24 \mathrm{~h}}$ ) with ultralente was about twice as large as the $22 \%$ with NPH $(p<0.05)$, but was intermediate with glargine at $31 \%(p=\mathrm{NS})$. By contrast, variability in the diurnal time-action profile (SD of diurnal day-to-day differences in GIR) for glargine was 30\% $(p<0.05)$ and $50 \%$ $(p<0.05)$ less than with NPH and ultralente, respectively. No serious adverse events were reported. Conclusions/ interpretation: Although representing insulins of different profiles, glargine and NPH showed a high and similar reproducibility of total absorption and glucodynamic effect, whereas ultralente proved to have poor reproducibility. However, while NPH yields peaks in concentration
\end{abstract}

H. E. Scholtz · S. G. Pretorius · D. H. Wessels · R. H. A. Becker Farmovs-Parexel Clinical Research Organisation,

Bloemfontein, South Africa

R. H. A. Becker $(\bowtie)$

Aventis Pharma Deutschland GmbH,

Industriepark Höchst/H 831,

65926 Frankfurt am Main, Germany

e-mail: reinhard.becker@sanofi-aventis.com

Tel.: +49-69-3054275

Fax: $+49-69-30580480$ and activity, glargine shows flat and non-fluctuating profiles resulting in less variation in day-to-day $24-\mathrm{h}$ activity.

Keywords Euglycaemic clamp · Glucodynamic · Insulin glargine $\cdot$ Insulin ultralente $\cdot$ NPH insulin .

Pharmacokinetic $\cdot$ Reproducibility

Abbreviations $\Delta$ : difference $\cdot \mathrm{C}_{\max }$ : maximum concentration - CUM: cumulative - GIR: glucose infusion rate $\cdot$ GIR-AUC ${ }_{0-24} \mathrm{~h}$ : area under the GIR-time curve up to $24 \mathrm{~h}$ - GIR $_{\max }$ : maximum GIR - GIR- $t_{50 \%}$ : time to $50 \%$ of GIR-AUC ${ }_{0-24 \mathrm{~h}} \cdot$ INS: insulin concentrations . $\mathrm{INS}-\mathrm{AUC}_{0-24 \mathrm{~h}}$ : area under the exogenous insulin concentration-time curve up to $24 \mathrm{~h} \cdot$ INS- $C_{\max }$ : maximum insulin concentrations $\cdot$ INS- $T_{\max }$ : times to INS- $C_{\max }$. LLOQ: lower limit of quantification - MSE: mean sum of the error terms - NPH: NPH insulin - $\mathrm{T}_{\max }$ : time to maximum concentration $\cdot$ ultralente: insulin ultralente

\section{Introduction}

Long-acting insulins, which serve to replace endogenous basal insulin release in patients with diabetes, need to provide predictable peakless time-concentration and timeaction profiles to mimic the slow, steady rate of insulin secreted in the fasted state. To this day, intermediate- and long-acting insulins, such as NPH insulin (NPH) and insulin ultralente (ultralente), are used as basal insulin therapy. Unfortunately, the pharmacokinetics of these traditional insulin preparations do not match the profiles of endogenous insulin secretion. In particular, intermediate-acting insulin shows a peak-action profile [1] and, as with ultralente, a huge day-to-day variability in absorption after s.c. injection [2]. These undesirable effects result in large fluctuations in systemic exposure, and through this unfavourable insulin action profiles. In particular, large intra-subject variability in onset, extent and duration of action of ultralente causes unpredictable metabolic control, including periods of hypo- and hyperglycaemia. The untimely action of NPH, by contrast, requires extra meals 
or snacks. Consequently, a major barrier to obtaining optimal glycaemic control in patients with diabetes results from their fear of iatrogenic hypoglycaemia caused by volatile fluctuations in the pharmacokinetics of common insulin $[3,4]$. Conversely, it is now well established that the hyperglycaemia occurring as a consequence of poor glycaemic control contributes to the development of microand possibly macrovascular complications $[5,6]$.

Insulin glargine, a novel, long-acting insulin, represents a true basal insulin with a predictable flat pharmacokinetic profile ensuring real clinical benefit. Several pharmacokinetic and pharmacodynamic studies characterised insulin glargine as an insulin with sustained, prolonged absorption [7], no pronounced peak, a near-24-h duration of action and a lower between-subject variability than NPH or ultralente [8]. A recent study with insulin detemir, another novel, long-acting insulin, compared the between-subject variability of insulin glargine, insulin detemir and NPH in patients with type 1 diabetes [9]. Here, we detail a study that compared the within-subject variability in systemic exposure and glucodynamic effects of insulin glargine with that of NPH and ultralente using the manual euglycaemic clamp technique in healthy volunteers. In addition, diurnal profile reproducibility was assessed [10].

\section{Subjects, materials and methods}

\section{Study design}

This trial was a single-dose, double-blind, randomised, parallel-group, replicate-design study investigating withinsubject variability in systemic exposure and glucodynamics of insulin glargine compared with NPH (Huminsulin basal 100) and ultralente (Ultralong) in healthy volunteers. Thirty-six healthy volunteers were allotted to three groups of 12 subjects per group. Each group received two consecutive injections of one of the study medications. Prior to the second injection, there was a wash-out period of at least 7 days. The study was performed at the Hoechst Marion Roussel Research Centre, Bloemfontein, South Africa, now Farmovs-Parexel.

\section{Study population}

Healthy, non-smoking, male volunteers aged $18-33$ years (mean 23.1 years), weighing between 66 and $100 \mathrm{~kg}$ (mean $79.6 \mathrm{~kg}$ ), with BMI values of $20.0-26.0 \mathrm{~kg} / \mathrm{m}^{2}$ (mean 23.5 $\mathrm{kg} / \mathrm{m}^{2}$ ), a normal oral glucose tolerance test and no clinically important abnormalities in their clinical chemistry, ECGs, vital signs and medical history or on physical examination were included in the study. The demographic and baseline characteristics of the study population were similar between groups: 18.3-29.3 years (mean 23.3), 18.9-32.5 years (mean 23.6) and 19.1-27.5 years (mean $22.3)$ in the insulin glargine $(n=12)$, NPH $(n=12)$ and ultralente $(n=12)$ groups, respectively. Body weight ranges for the insulin glargine, NPH and ultralente groups were
67.1-97.2 kg (mean 77.9), 69.0-91.2 kg (mean 80.5) and 65.6-99.8 kg (mean 80.4), respectively. The BMI ranges were $20.0-25.8 \mathrm{~kg} / \mathrm{m}^{2}$ (mean 22.8), 21.1-26.0 kg/m (mean 23.9) and $22.1-26.0 \mathrm{~kg} / \mathrm{m}^{2}$ (mean 23.7), respectively. All volunteers provided written, informed consent prior to initiation of the investigation; the study was carried out in accordance with the Declaration of Helsinki.

\section{Study medication}

Each volunteer received two consecutive ( $\geq 7$-day washout) s.c. injections of insulin glargine (12 subjects), NPH (12 subjects) or ultralente (12 subjects) at a dose of $0.4 \mathrm{IU} /$ $\mathrm{kg}$ body weight administered by a physician or nurse otherwise not involved in the study. The peri-umbilical abdominal area was chosen for injection with a $0.13 \times 12$ $\mathrm{mm}$ single-use syringe with integrated needle (Braun Omnican) for comparison with previous single-dose studies.

\section{Clamp procedure}

Subjects remained fasting from 22.00 hours the night before to the end of the entire study procedure. The injection time defined the time zero of the insulin action period, which was monitored for $24 \mathrm{~h}$. Glucose infusion rate (GIR), blood glucose concentration, serum immunoreactive insulin and serum $\mathrm{C}$-peptide concentrations were recorded for pharmacokinetic and pharmacodynamic evaluations. Blood samples were taken from an i.v. cannula, which was inserted into the hand or wrist vein, for measurement of blood glucose, serum insulin and serum C-peptide levels.

Blood glucose concentrations were measured every 10 min after administration of the study medication with a Yellow Springs Instruments 2300S Glucose Analyzer (Yellow Springs Instruments, Yellow Springs, OH, USA) using the glucose oxidase method. Baseline blood glucose concentration was calculated as the mean value of blood glucose measurements taken at 60,30, 15 and $5 \mathrm{~min}$ prior to study medication. A drop in blood glucose up to a maximum of $10 \%$ from baseline signified the initiation of the glucose infusion. A $20 \%$ glucose solution was infused at a manually stepwise-adjusted variable rate to restore and maintain the subject's baseline blood glucose concentration.

Pharmacokinetic data collection Serum total immunoreactive insulin was measured at 30,15 and 5 min prior to and every $60 \mathrm{~min}$ up to $24 \mathrm{~h}$ after administration of the study medication using an RIA for human insulin with an in vitro cross-reactivity with insulin glargine of about $50 \%$. The lower limit of quantification (LLOQ) was 2.5 $\mu \mathrm{IU} / \mathrm{ml}$. Simultaneous serum C-peptide concentrations were also determined with an RIA with an LLOQ of 0.15 $\mathrm{ng} / \mathrm{ml}$ (Analytical Services Division, Farmovs Research Centre, Bloemfontein, South Africa). Immunoreactive insu- 
lin concentrations (INS) were corrected for endogenous insulin using serum C-peptide levels to yield exogenous insulin concentration profiles and to observe maximum concentrations (INS- $C_{\max }$ ), times to INS- $C_{\max }$ (INS- $T_{\max }$ ) and to calculate the area under the exogenous insulin concentration-time curve up to $24 \mathrm{~h}$ (INS-AUC $\mathrm{IN}_{0-24 \mathrm{~h}}$ ).

Pharmacodynamic data collection The time-action profile was characterised by the area under the GIR-time curve up to $24 \mathrm{~h}\left(\mathrm{GIR}-\mathrm{AUC}_{0-24 \mathrm{~h}}\right)$, by the maximum GIR $\left(\mathrm{GIR}_{\max }\right)$, time to $50 \%$ of GIR-AUC ${ }_{0-24 \mathrm{~h}}\left(\mathrm{GIR}-t_{50 \%}\right)$ and the time to $\mathrm{GIR}_{\max }\left(\mathrm{GIR}-T_{\max }\right)$.

\section{Safety data}

Adverse events were reported by the subject or noted by the investigator. Routine laboratory tests included haematology, clinical chemistry and urinalysis. Determination of human insulin antibodies, a physical examination and a 12lead ECG were also carried out.

\section{Statistical methods}

Pharmacokinetics For insulin, area under the concentration-time curve $\left(\mathrm{AUC}_{0-24 \mathrm{~h}}\right)$ was calculated according to the linear trapezoidal rule up to $24 \mathrm{~h}$ after injection of the study medication. INS- $C_{\max }$ was read directly from the derived serum concentrations of exogenous insulin. ANOVA for treatment and subject effect was applied on natural logarithmic (ln)-transformed data. Antilog point estimates with $90 \%$ CIs were obtained for the mean ratio 'Clamp 2-Clamp 1' on the ln-scale with period and subject effect per insulin, and for the respective ratios of treatment means (secondary analysis). Non-parametric analysis was performed and $90 \%$ CIs calculated for INS- $T_{\max }$ according to Steinijans and Diletti.

Pharmacodynamics For glucose, area under the GIR-time curve was calculated as the exact area under the stepwise constant function for the respective time intervals of the 24-h GIR-time profile; the GIR- $t_{50 \%}$ was extrapolated from this. The determination of $\mathrm{GIR}_{\max }$ was based on a 'smoothed three-point running mean' GIR curve for each subject. For each measured value of GIR, a mean GIR value was calculated from the previous, actual and following GIR values (this corresponded to mean values of 20-min intervals from injection of the study medication up to the end of the clamp period). The GIR max $_{\text {was }}$ read directly from the smoothed GIR-time curves and the times of $\mathrm{GIR}_{\max }$ were reported as the blood sampling times corresponding with the $\mathrm{GIR}_{\max }$. ANOVA of untransformed data was applied to the area under the smoothed (three-point running means smoother) GIR curve over $24 \mathrm{~h}$ $\left(\right.$ GIR-AUC $\left.{ }_{0-24} \mathrm{~h}\right), \mathrm{GIR}_{-} t_{50 \%}$ and $\mathrm{GIR}_{\max }$, and CIs calculated based on Fieller's theorem. Non-parametric analysis was performed and $90 \%$ CIs calculated for GIR- $T_{\max }$.
Between-treatment comparisons were conducted as described above.

Variability in pharmacokinetics and pharmacodynamics Within-subject variability was assessed as intra-individual $\mathrm{CV}$ values, taken from the mean sum of the error terms (MSE) as calculated by the ANOVA on untransformed values for GIR-AUC ${ }_{0-24} \mathrm{~h}, \mathrm{GIR}_{\max }$, GIR- $t_{50 \%}$ and INS$T_{\max }$, and on ln-transformed values for INS-AUC and INS$C_{\max } . \quad(\mathrm{CV} \%$ of untransformed data=SQRT[MSE $]$ LSM $\times 100 ; \quad C V \%$ of ln-transformed data=SQRT[(EXP (MSE)-1] $\times 100$.) For comparison of variances between treatments, the statistical $F$-distribution was used to compute $90 \%$ CIs on the ratio of the two variances.

Profile reproducibility Profile reproducibility was assessed in two steps: by the absolute individual cumulative (CUM) between-day differences in insulin concentration $\left(\Delta_{\text {absolute }}-\right.$ INS-CUM) and GIR profiles $\left(\Delta_{\text {absolute }}-\right.$ GIRCUM) and by individual SD values of hourly between-day differences (untransformed, raw data) in insulin concentrations $\left(\mathrm{SD}-\Delta_{\text {raw }}-\mathrm{INS}\right)$ and GIR $\left(\mathrm{SD}-\Delta_{\text {raw }}-\mathrm{GIR}\right)$ over $24 \mathrm{~h}$. For comparison between treatments, non-parametric analysis was performed for these metrics. A stem-andleaf plot for outlier detection was also accomplished. An additional comparison of $\Delta_{\text {absolute }}-$ INS-CUM was performed with insulin glargine concentrations corrected for the observed underestimation relative to NPH (i.e. equivalence in INS-AUC ${ }_{0-24 \mathrm{~h}}$ was assumed).

\section{Results}

Clamp performance The blood glucose concentration was restored to each individual's fasting blood glucose concentration after study medication and maintained during the entire clamp. The mean within-subject CV of the blood glucose concentration was: (1) after insulin glargine: $4.1 \%$ (Visit 2), 4.0\% (Visit 3); (2) after NPH: 4.7\% (Visit 2), 4.4\% (Visit 3); and (3) after ultralente: 4.2\% (Visit 2), $4.1 \%$ (Visit 3). These CVs are similar to those reported for closed-loop feedback-controlled euglycaemic clamps [11]. There were no relevant differences in mean baseline blood glucose concentrations between visits or treatment groups before administration of the study medication (insulin glargine: $4.5 \mathrm{mmol} / 1$ [Visit 2], $4.5 \mathrm{mmol} / 1$ [Visit 3]; NPH: $4.5 \mathrm{mmol} / 1$ [Visit 2], $4.5 \mathrm{mmol} / 1$ [Visit 3]; ultralente: 4.5 $\mathrm{mmol} / 1$ [Visit 2], $4.5 \mathrm{mmol} / 1$ [Visit 3]).

Pharmacokinetic parameters Insulin glargine presented matching serum concentration curves in 10 of 12 subjects. Two subjects were identified with poorly superimposable profiles, but were nevertheless included for analysis. Ultralente presented with distinctly separate serum concentration curves in the majority of subjects, while NPH concentration profiles were fairly reproduced in each individual. As a consequence, insulin glargine and NPH each showed superimposable median profiles, while 

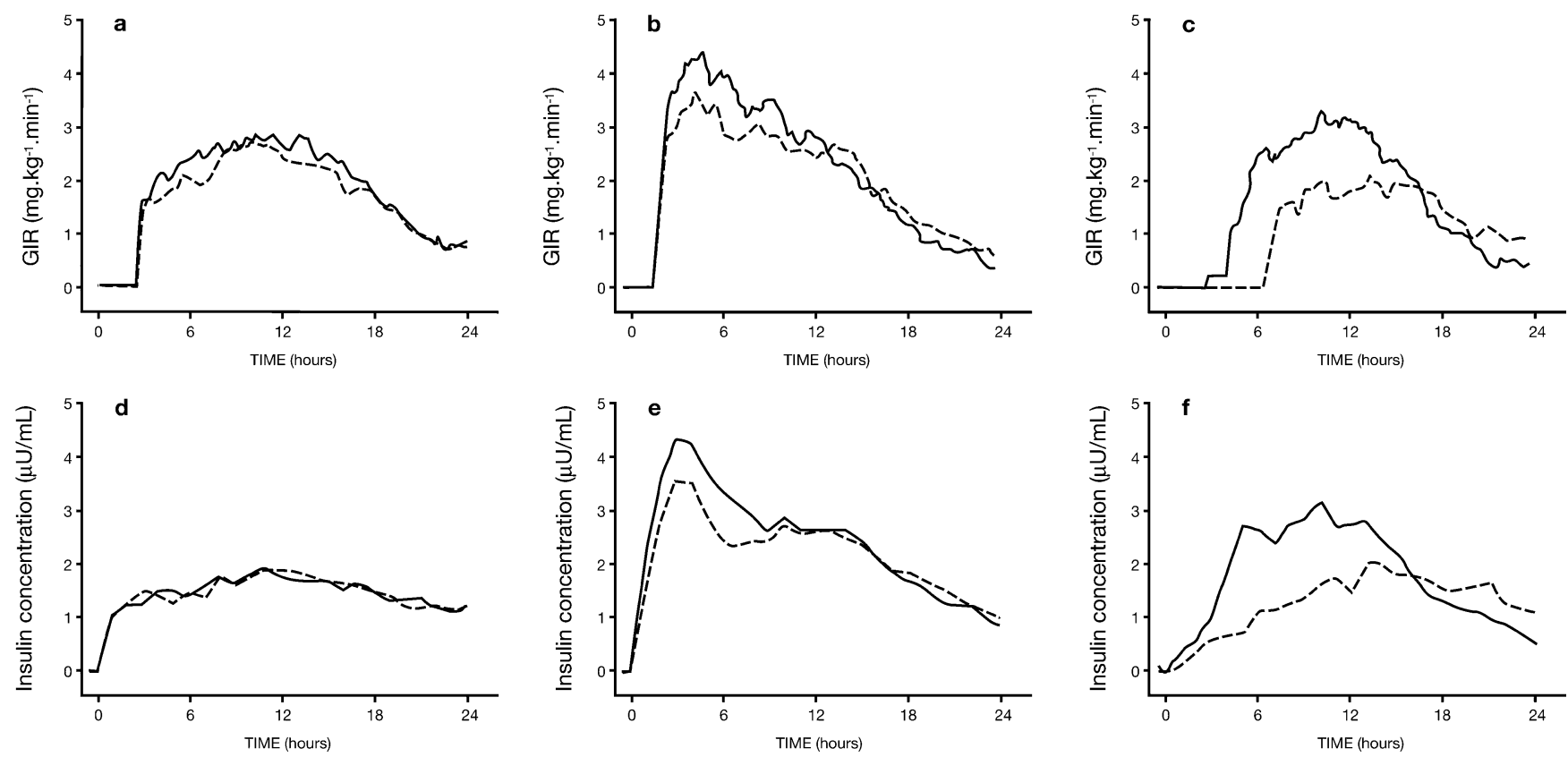

Fig. 1 a-c Pharmacodynamics (average glucose infusion rate $[G I R] ; \mathrm{mg} \cdot \mathrm{kg}^{-1} \cdot \mathrm{min}^{-1}$, median) for insulin glargine (a), NPH insulin (NPH) (b) and insulin ultralente (ultralente) (c); median GIR values of Clamp 1 (solid line) and (ultralente) Clamp 2 (dashed line) are given. d-f Pharmacokinetics (exogenous immunoreactive median serum insulin concentrations, estimated from serum immunoreactive

ultralente presented with a day-to-day difference of, on average, $3 \mathrm{~h}$ in time (INS- $T_{\max }$ ) to similar maximum concentrations (INS- $C_{\text {max }}$ ).

The averaged total systemic insulin exposure (INS$\mathrm{AUC}_{0-24 \mathrm{~h}}$ ) was assayed approximately $40 \%$ higher with NPH than with ultralente and insulin glargine, which were equal. INS- $C_{\max }$ after NPH was observed on average $4 \mathrm{~h}$ (median) after injection, which was significantly earlier than the $12 \mathrm{~h}$ after insulin glargine and the $13 \mathrm{~h}$ after ultralente (Fig. 1a), despite a wide range of INS- $T_{\max }$ occurrences of the latter insulins (Tables 1 and 2).

Pharmacodynamic values The average 24-h glucose disposal (GIR-AUC ${ }_{0-24} \mathrm{~h}$ ) was similar with insulin glargine and NPH. It was slightly less with ultralente as compared insulin and serum C-peptide; $\mu \mathrm{U} / \mathrm{ml}$ ) for insulin glargine (d), NPH (e) and ultralente (f); median insulin concentrations of Clamp 1 (solid line) and Clamp 2 (dashed line) are given. The figure shows that generally lower and non-fluctuating serum insulin concentrations, as compared with NPH, were attained for insulin glargine, while ultralente displayed large day-to-day variability

with both insulin glargine and NPH, although the wide margins did not allow for statistical inferences. Insulin glargine and ultralente displayed half of their activity within, on average, 11.5 and $12.5 \mathrm{~h}$, respectively, as compared with $9.5 \mathrm{~h}$ with NPH (i.e. within significantly less time; Tables 3 and 4). Very wide ranges of late GIR- $T_{\max }$ at low $\mathrm{GIR}_{\max }$ were observed for insulin glargine and ultralente and distinctly smaller ranges of early GIR- $T_{\max }$ at larger $\mathrm{GIR}_{\max }$ for NPH.

Within-subject variabilities of pharmacokinetics and pharmacodynamics The systemic insulin exposure after ultralente was characterised by fundamentally higher day-today (within-subject) variability in core parameters than after insulin glargine or NPH (Fig. 1b). For INS-AUC ${ }_{0-24} \mathrm{~h}$,

Table 1 Replicate pharmacokinetics of insulin glargine, NPH insulin and insulin ultralente parameters

\begin{tabular}{|c|c|c|c|c|}
\hline & \multirow[t]{2}{*}{ Study day } & \multicolumn{3}{|c|}{ Parameter, mean (SD) } \\
\hline & & Insulin glargine $^{\mathrm{a}}$ & NPH insulin & Insulin ultralente \\
\hline \multirow[t]{2}{*}{$\mathrm{INS}-\mathrm{AUC}_{0-24 \mathrm{~h}}\left(\mu \mathrm{I} \cdot \mathrm{U} \cdot \mathrm{hml}^{-1}\right)$} & Clamp 1 & $169(39)$ & $300(59)$ & $205(106)$ \\
\hline & Clamp 2 & $189(52)$ & $257(52)$ & $176(103)$ \\
\hline \multirow[t]{2}{*}{$\mathrm{INS}-C_{\max }(\mu \mathrm{IU} / \mathrm{ml})$} & Clamp 1 & $10.0(2.5)$ & $23.2(5.0)$ & $16.4(8.3)$ \\
\hline & Clamp 2 & $12.1(5.7)$ & $18.4(2.3)$ & $14.3(8.4)$ \\
\hline \multirow[t]{2}{*}{$\mathrm{INS}-T_{\max }(\mathrm{h})$} & Clamp 1 & $12.4(4.5)$ & $3.9(1.4)$ & $10.8(5.5)$ \\
\hline & Clamp 2 & $11.0(5.4)$ & $4.7(3.0)$ & $13.6(6.0)$ \\
\hline
\end{tabular}

INS insulin, $C_{\max }$ maximum concentration, $T_{\max }$ time to $C_{\max }$

${ }^{a}$ Insulin glargine concentrations are not corrected for differences in cross-reactivity with human insulin (see Pharmacokinetic data collection) 
Table 2 Comparison of pharmacokinetics between treatments: point estimates ( $\%$ [90\% CI] $)$

\begin{tabular}{|c|c|c|c|}
\hline & Insulin glargine ${ }^{\mathrm{a}} / \mathrm{NPH}$ insulin & Insulin glargine ${ }^{\mathrm{a}} /$ insulin ultralente & NPH insulin/insulin ultralente \\
\hline $\mathrm{INS}-\mathrm{AUC}_{0-24 \mathrm{~h}}$ & $64(53,77)^{\mathrm{b}}$ & $89(74,108)$ & $140(116,170)^{b}$ \\
\hline INS $-C_{\max }$ & $52(42,63)^{b}$ & $68(56,84)^{b}$ & $132(108,162)^{b}$ \\
\hline Median INS $-T_{\max }(\mathrm{h})$ & $8(6,9)^{\mathrm{b}}$ & $-1(-4,2)$ & $-8(-11,-6)^{\mathrm{b}}$ \\
\hline
\end{tabular}

INS insulin, $C_{\max }$ maximum concentration, $T_{\max }$ time to $C_{\max }$

${ }^{a}$ Insulin glargine concentrations are not corrected for differences in cross-reactivity with human insulin (see Pharmacokinetic data collection)

${ }^{\mathrm{b}} p<0.05$

insulin glargine and NPH yielded low within-subject CVs, compared with ultralente. Both insulin glargine and NPH also showed a lower INS- $C_{\max }$ within-subject variability compared with ultralente. In line with the flat profile, the $\mathrm{CV} \%$ in INS- $T_{\max }$ was larger after insulin glargine than after either NPH or ultralente (Table 5).

The within-subject GIR variability for GIR-AUC ${ }_{0-24} \mathrm{~h}$ was about twice as large with ultralente as NPH, and intermediate for insulin glargine. The $\mathrm{CV} \%$ for GIR- $t_{50} \%$ was low and about the same for insulin glargine and NPH, but almost three times as large for ultralente. A similar $\mathrm{CV} \%$ in $\mathrm{GIR}_{\max }$ was seen for NPH and insulin glargine, but was larger for ultralente. In any event, there were no statistical differences in variances for any parameter between insulin glargine and $\mathrm{NPH}$, while variances with ultralente were significantly larger for INS- $\mathrm{AUC}_{0-24} \mathrm{~h}$, INS- $C_{\max }$ and GIR- $t_{50 \%}$ compared with insulin glargine and $\mathrm{NPH}$, and for GIR-AUC $0-24 \mathrm{~h}$ compared with NPH (Table 5).

Profile reproducibility The individual absolute cumulative between-day differences in insulin concentration $\left(\Delta_{\text {absolute }}-\right.$ INS-CUM) were similar with insulin glargine and $\mathrm{NPH}$, with and without adjustment for the imbalance in INS-AUC $0-24 \mathrm{~h}$. Absolute individual between-day differences were significantly less for insulin glargine than after ultralente. The variation in the hourly between-day differences, expressed as individual SD in insulin concentrations (SD- $\Delta_{\text {raw }}-$ INS), was less for insulin glargine profiles than for NPH (similar when adjusted for the imbalance), and significantly less than for ultralente (Fig. 2). Accordingly, the absolute between-day differences in the time-action profiles $\left(\Delta_{\text {absolute}}-\right.$ GIR-CUM) were similar with insulin glargine and NPH, but less compared with ultralente. The variation in the between-day differences (SD- $\Delta_{\text {raw }}-$ GIR) with insulin glargine, however, was 30\% less compared with NPH and 50\% less compared with ultralente (Fig. 2). Moreover, both results were statistically significant when two extreme values in the insulin glargine group were excluded, as indicated by a stem-and-leaf plot analysis (Table 6).

C-peptide Suppression of endogenous insulin secretion assessed by a reduction in C-peptide concentration was slightly more rapid with NPH than with insulin glargine or ultralente, but was uniformly the same at $>50 \%$ after the
Table 3 Replicate glucodynamics of insulin glargine, NPH insulin and insulin ultralente

\begin{tabular}{lllcc}
\hline & Study day & \multicolumn{3}{l}{ Parameter, mean (SD or range) } \\
\cline { 3 - 5 } & & Insulin glargine & NPH insulin & Insulin ultralente \\
\hline GIR-AUC $0-24 \mathrm{~h}(\mathrm{mg} / \mathrm{kg})$ & Clamp 1 & $2,558(1,400)$ & $2,993(1,091)$ & $2,593(1,313)$ \\
& Clamp 2 & $2,987(1,820)$ & $2,847(1,133)$ & $2,025(1,360)$ \\
GIR- $t_{50 \%}(\mathrm{~h})$ & Clamp 1 & $12(2)$ & $9(1)$ & $11(2)$ \\
$\mathrm{GIR}_{\max }\left(\mathrm{mg} \cdot \mathrm{kg}^{-1} \cdot \mathrm{min}^{-1}\right)$ & Clamp 2 & $11(2)$ & $10(1)$ & $15(3)$ \\
& Clamp 1 & $3.2(1.3)$ & $5.0(1.7)$ & $4.1(2.0)$ \\
Median GIR- $T_{\max }, \mathrm{h}($ range) & Clamp 2 & $3.7(2.7)$ & $4.4(1.8)$ & $3.3(2.0)$ \\
& Clamp 1 & $8.6(4.4-11.7)$ & $4.6(2.0-10.0)$ & $8.1(4.5-15.4)$ \\
& Clamp 2 & $10.1(4.0-22.2)$ & $4.9(3.5-13.7)$ & $13.1(5.5-23.5)$ \\
\hline
\end{tabular}

GIR glucose infusion rate, $T_{\max }$ time to $\mathrm{C}_{\max }, G I R-t_{50 \%}$ time to $50 \%$ of GIR-AUC ${ }_{0-24} \mathrm{~h}$

Table 4 Comparison of glucodynamics between treatments: point estimates (\% $[90 \% \mathrm{CI}])$

\begin{tabular}{lccc}
\hline & Insulin glargine/NPH insulin & Insulin glargine/insulin ultralente & NPH insulin/insulin ultralente \\
\hline GIR-AUC $_{0-24 \mathrm{~h}}$ & $93(67,128)$ & $120(83,179)$ & $129(97,176)$ \\
$\mathrm{GIR}_{-} t_{50 \%}$ & $125(118,132)^{\mathrm{a}}$ & $92(83,100)$ & $73(65,82)^{\mathrm{a}}$ \\
$\mathrm{GIR}_{\max }$ & $72(52,97)^{\mathrm{a}}$ & $93(64,133)$ & $129(97,174)$ \\
Median GIR- $_{\max }(\mathrm{h})$ & $3.6(2.1,5.3)^{\mathrm{a}}$ & $-1.9(-4.1,0.8)$ & $-5.7(-7.8,-3.0)^{\mathrm{a}}$ \\
\hline
\end{tabular}

GIR glucose infusion rate, GIR $\max$ maximum GIR, $T_{\max }$ time to $\mathrm{GIR}_{\max }, G I R-t_{50 \%}$ time to $50 \%$ of GIR-AUC ${ }_{0-24} \mathrm{~h}$ ${ }^{\mathrm{a}} p<0.05$ 
Table 5 Within-subject variability: CV\% (95\% CI)

\begin{tabular}{|c|c|c|c|}
\hline & Insulin glargine & NPH insulin & Insulin ultralente \\
\hline INS-AUC $0-24 \mathrm{~h}$ & $15(8,22)^{\mathrm{a}}$ & $19(11,28)^{\mathrm{b}}$ & $67(35,99)$ \\
\hline INS- $C_{\max }$ & $29(16,41)^{\mathrm{a}}$ & $21(12,31)^{b}$ & $64(34,95)$ \\
\hline $\mathrm{INS}-T_{\max }$ & $48(26,69)$ & $36(20,52)$ & $37(20,55)$ \\
\hline $\mathrm{GIR}-\mathrm{AUC}_{0-24 \mathrm{~h}}$ & $31(17,45)$ & $22(12,32)^{b}$ & $49(26,72)$ \\
\hline $\mathrm{GIR}_{\max }$ & $27(15,38)$ & $23(13,33)$ & $38(20,57)$ \\
\hline GIR- $t_{50 \%}$ & $13(7,19)^{\mathrm{a}}$ & $11(6,17)^{b}$ & $32(17,47)$ \\
\hline
\end{tabular}

$C V$ (ANOVA) taken from the mean sum of the error terms, INS insulin, $C_{\max }$ maximum concentration, $T_{\max }$ time to $C_{\max }, G I R_{\max }$ maximum glucose infusion rate, GIR- $t_{50 \%}$ time to $50 \%$ of GIR$\mathrm{AUC}_{0-24 \mathrm{~h}}$

${ }_{p}^{a}<<0.05$, insulin glargine vs insulin ultralente

${ }^{\mathrm{b}} p<0.05$, NPH insulin vs insulin ultralente

6th hour with each of the insulins (mean change from baseline: insulin glargine, $-67 \%$ vs $-62 \%$; NPH, $-66 \%$ vs $-64 \%$; ultralente, $-65 \%$ vs $-66 \%$; all Clamp 1 vs Clamp 2).

Safety No serious adverse events were reported. Adverse events, reported in 19 subjects, were most frequently mildto-moderate headaches, which occurred in similar numbers between the groups, and iron-deficiency anaemia (six subjects). Both events were related to the clamp procedure.

\section{Discussion}

This study assessed the day-to-day variability in the timeconcentration and time-action profiles of insulin glargine compared with NPH and ultralente, employing the manual euglycaemic clamp technique in healthy volunteers. Euglycaemia was reliably established with blood glucose concentrations varying by $<5 \%$ within each subject's clamp period, with C-peptide suppression as a surrogate for the reduction of endogenous insulin release, which was uniformly the same in all clamps from the 6th hour onwards. In the present study, insulin was injected into the abdomen rather than the thigh. Although the abdomen is no longer commonly used as an injection site we do not believe that this procedure influenced the results, as supported by others [7].
Table 6 Profile reproducibility: ratio of medians

\begin{tabular}{lll}
\hline & $\begin{array}{l}\text { Insulin glargine/NPH } \\
\text { insulin }\end{array}$ & $\begin{array}{l}\text { Insulin glargine/insulin } \\
\text { ultralente }\end{array}$ \\
\hline$\Delta_{\text {absolute }}$ INS- & $\begin{array}{l}0.83\left(0.73^{\mathrm{c}}\right) / 1.29^{\mathrm{a}} \\
\left(1.14^{\mathrm{a}, \mathrm{c}}\right)\end{array}$ & $0.41^{\mathrm{b}}\left(0.37^{\mathrm{b}, \mathrm{c}}\right)$ \\
CUM & $0.95\left(0.84^{\mathrm{c}}\right)$ & $0.60^{\mathrm{b}}\left(0.54^{\mathrm{b}, \mathrm{c}}\right)$ \\
$\Delta_{\text {absolute }}$ GIR- & & \\
CUM & & $0.49^{\mathrm{b}}\left(0.43^{\mathrm{b}, \mathrm{c}}\right)$ \\
SD- $\Delta_{\text {raw }}-\mathrm{INS}$ & $\begin{array}{l}0.67^{\mathrm{b}}\left(0.58^{\mathrm{b}, \mathrm{c}}\right) / 1.04^{\mathrm{a}} \\
\left(0.90^{\mathrm{a}, \mathrm{c}}\right)\end{array}$ & \\
SD- $\Delta_{\text {raw }}-\mathrm{GIR}$ & $0.70^{\mathrm{b}}\left(0.66^{\mathrm{b}, \mathrm{c}}\right)$ & $0.48^{\mathrm{b}}\left(0.46^{\mathrm{b}, \mathrm{c}}\right)$ \\
\hline
\end{tabular}

$\Delta$ difference, INS insulin, CUM cumulative, GIR glucose infusion rate

${ }^{\mathrm{a}}$ Normalised for INS-AUC ${ }_{0-24 \mathrm{~h}}$

${ }^{\mathrm{b}} p<0.05$

${ }^{c}$ Excluding outliers

There were clear differences in the time-concentration profiles of the three insulins. Although both insulin glargine and NPH displayed low variability in systemic exposure, injection of NPH produced defined early peak exposures. Ultralente, which in general affords a flat profile, was associated with an ill-defined onset in absorption and, consequently, large variability in systemic exposure.

These insulin concentration profiles are fully corroborated by the glucodynamic profiles. Insulin glargine demonstrated a very reproducible activity profile with no pronounced peak and an early, modest onset in glucose lowering, requiring a soft onset in equalising glucose infusion to commence within $2-3 \mathrm{~h}$ and be sustained for $24 \mathrm{~h}$. $\mathrm{NPH}$, by contrast, required a more robust glucose infusion to commence prior to the second hour after injection and thus generated a defined peak in glucose supply at the fifth hour, after which it declined. Ultralente actually presented two markedly different profiles with a highly variable onset in both glucose lowering and corresponding glucose infusion, although it eventually displayed a profile with no pronounced peak.

Concerning assessment of within-subject variability in glucodynamics, there are limitations on euglycaemic clamps of basal insulins, and on manual clamps in nondiabetic subjects in particular [12]. The euglycaemic-hyper-
Fig. 2 Distribution of SD of diurnal individual day-to-day differences in insulin concentration (a) and glucose infusion rate $(G I R ; \mathbf{b})$ a

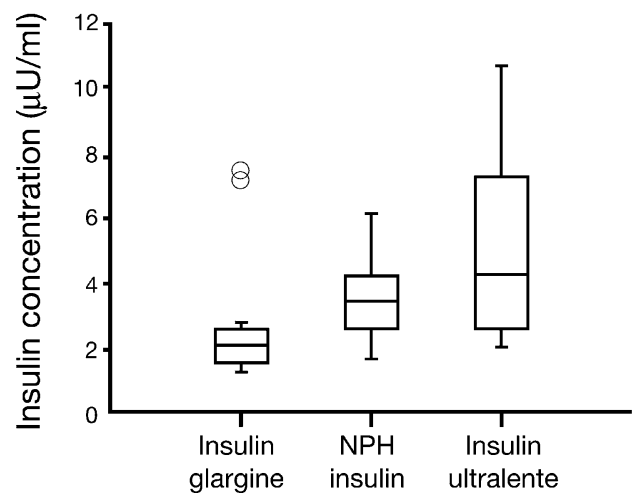

b

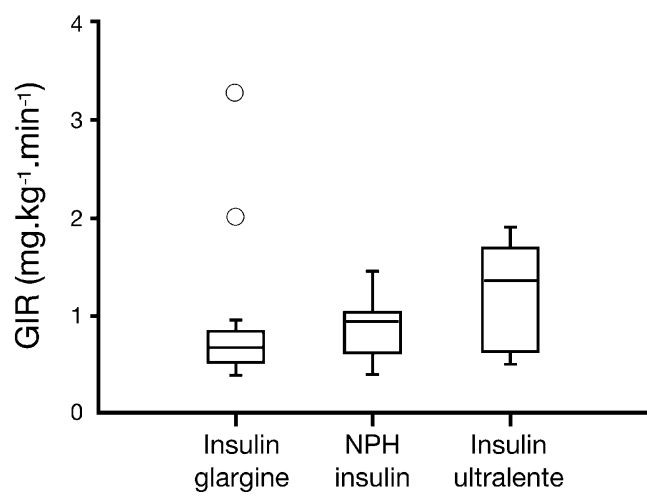


insulinaemic clamp technique was developed to examine insulin sensitivity, which requires elevated steady-state insulin concentrations $[11,13]$. However, glucose disposal in lasting euglycaemic-hyperinsulinaemic clamps becomes subject to increasing insulin sensitivity and non-oxidative glucose uptake [14]. Therefore, in line with the complex experimental interplay between a required reduction in blood glucose concentration of up to $10 \%$ that triggers the counter-glucose infusion and that, thus, follows the genuine onset of glucose-lowering activity, it is not so much the inherent pharmacokinetics of a basal insulin, but rather the sluggish onset of the manual adjustment of this infusion that determines the early variability in GIR. As a result, over the complete 24-h period after injection, the withinsubject variability of the GIR parameters was lowest when the insulin effect was strongest, which was seen with NPH. The large variability of ultralente action, by contrast, is predominantly due to the erratic onset in absorption and, in turn, action.

The more recent investigation of insulin detemir, $\mathrm{NPH}$ and insulin glargine, all intended for basal insulin replacement, using a Biostator-based euglycaemic clamp in patients with type 1 diabetes, is in line with this notion [9]. Tight blood glucose control is at the expense of larger fluctuation in Biostator-adjusted glucose infusion, which in turn requires vigorous smoothing of GIR readings to generate similar smooth biological response profiles as compared with manually adjusted clamps [15]. The more robust onset of action after insulin detemir resulting in less fluctuation in glucose infusion may explain, at least in part, the perceived less variable profile as compared with insulin glargine.

Overall, the common summary parameters do not appropriately reflect the actual day-to-day variation in fluctuation in concentration or in activity over $24 \mathrm{~h}$, which are of interest to a patient using a basal insulin. To this end, the profile reproducibility was assessed. Although the direct comparison of insulin glargine and NPH for variations in concentration was limited by the presumed bioanalytical underestimation of insulin glargine, insulin glargine presented at least equal profile reproducibility to $\mathrm{NPH}$, even when adjusted to equivalence in systemic exposure to NPH, and superiority to ultralente. More importantly, superiority in reproducibility was visible in the glucodynamic profile, where insulin glargine presented $30-50 \%$ less variation over $24 \mathrm{~h}$ compared with NPH and ultralente, respectively.

The flat time-concentration profile seen in healthy volunteers under controlled conditions was confirmed to be well reproducible in patients with type 1 diabetes at steady-state [16]. Therefore, from a clinical viewpoint, since hypoglycaemia is one of the most common and feared side-effects of insulin therapy [4], the use of preparations such as insulin glargine, which have a smooth 24-h profile and low within-patient variability, is favourable and may encourage greater adherence to insulin therapy. Insulin glargine is associated with a lower incidence of nocturnal hypoglycaemia compared with NPH $[17,18]$, and this could be due to the relatively flat action profile of insulin glargine compared with NPH. Further, the more opportune activity profile of insulin glargine facilitates treating to target $\mathrm{HbA}_{1} \mathrm{c}$ levels with a lower incidence of hypoglycaemia. Indeed, it has already been demonstrated that insulin glargine enables treating to a target $\mathrm{HbA}_{1} \mathrm{c}$ of $<7 \%$ [19]. In addition, when treated to a comparable $\mathrm{HbA}_{1} \mathrm{c}$ target, patients receiving insulin glargine experience a significantly lower incidence of severe hypoglycaemia compared with patients receiving NPH [20].

In summary, although representing insulins of different action profiles, under experimental conditions in manual euglycaemic clamps in healthy volunteers, the reproducibility of time-concentration and glucodynamic effects was high and favourable for insulin glargine and $\mathrm{NPH}$, but low for ultralente. Insulin glargine generally shows low day-today, within-subject variations in serum insulin concentration and the corresponding glucodynamic effect, which implies improved reproducibility of basal glucose control with insulin glargine.

Acknowledgements This study was supported by Aventis Pharmaceuticals, a company of the sanofi-aventis Group. Data from this manuscript have been previously presented in Abstract form at the EASD and ADA meetings in 2004.

Duality of interest

R.H.A. Becker is an employee of Aventis Pharma, a company of the sanofi-aventis group.

\section{References}

1. Bolli GB (1989) The pharmacokinetic basis of insulin therapy in diabetes mellitus. Diabetes Res Clin Pract 6:S3-S16

2. Binder C, Lauritzen T, Faber O, Pramming S (1984) Insulin pharmacokinetics. Diabetes Care 7:188-199

3. Cryer P, Childs B (2002) Negotiating the barrier of hypoglycemia in diabetes. Diabetes Spectrum 15:20-27

4. Cryer P (1999) Hypoglycemia is the limiting factor in the management of diabetes. Diabetes Metab Res Rev 15:42-46

5. DCCT (1993) The effect of intensive treatment of diabetes on the development and progression of long-term complications in insulin-dependent diabetes mellitus. The Diabetes Control and Complications Trial Research Group. N Engl J Med 329:977986

6. UKPDS (1998) Intensive blood-glucose control with sulphonylureas or insulin compared with conventional treatment and risk of complications in patients with type 2 diabetes (UKPDS 33). (UK Prospective Diabetes Study Group). Lancet 352:837853

7. Owens D, Coates P, Luzio S, Tinbergen J, Kurzhals R (2000) Pharmacokinetics of [125]I-labelled insulin glargine (HOE 901) in healthy men. Diabetes Care 23:813-819

8. Lepore M, Pampanelli S, Fanelli C et al (2000) Pharmacokinetics and pharmacodynamics of subcutaneous injection of long-acting human insulin analog glargine, NPH insulin, and ultralente human insulin and continuous subcutaneous infusion of insulin lispro. Diabetes 49:2142-2148

9. Heise T, Nosek L, Ronn BB et al (2004) Lower within-subject variability of insulin detemir in comparison to NPH insulin and insulin glargine in people with type 1 diabetes. Diabetes 53:1614-1620

10. Scholtz H, Becker R (2004) Reproducibility of serum insulin and glucose infusion rate profiles of insulin glargine compared with NPH insulin and insulin ultralente. Diabetologia 47 [Suppl 1]:A273 (Abstract 753) 
11. DeFronzo R, Tobin JD, Andres R (1979) Glucose clamp technique: a method for quantifying insulin secretion and resistance. Am J Physiol 237:E214-E223

12. Ponchner M, Heine RJ, Pernet A et al (1984) A comparison of the artificial pancreas (glucose controlled insulin infusion system) and a manual technique for assessing insulin sensitivity during euglycaemic clamping. Diabetologia 26:420-425

13. Bokemark L, Froden A, Attval S, Wikstrand J, Fagerberg B (2000) The euglycaemic hyperinsulinemic clamp examination: variability and reproducibility. Scand J Clin Invest 60:27-36

14. Bergman RN, Hope ID, Yang YJ et al (1989) Assessment of insulin sensitivity in vivo: a critical review. Diabetes Metab Rev 5:411-429

15. Becker RHA, Frick A, Saoud JB et al (2005) Variability in concentration and glucodynamic profiles of insulin glargine in healthy volunteers. Diabetes 54 [Suppl 1]:A145 (Abstract 586)

16. Heise T, Bott S, Rave K, Dressler A, Rosskamp R, Heinemann L (2002) No evidence for accumulation of insulin glargine (LANTUS): a multiple injection study in patients with Type 1 diabetes. Diabet Med 19:490-495
17. Yki-Jarvinen H, Dressler A, Ziemen M (2000) Less nocturnal hypoglycemia and better post-dinner glucose control with bedtime insulin glargine compared with bedtime NPH insulin during insulin combination therapy in type 2 diabetes. HOE 901/3002 Study Group. Diabetes Care 23:1130-1136

18. Ratner RE, Hirsch IB, Neifing JL, Garg SK, Mecca TE, Wilson CA (2000) Less hypoglycemia with insulin glargine in intensive insulin therapy for Type 1 diabetes. Diabetes Care 23:639-643

19. Riddle M, Rosenstock J, Gerich J; Insulin Glargine 4002 Study Investigators (2003) The Treat-to-Target Trial: randomized addition of glargine or human NPH insulin to oral therapy of type 2 diabetic patients. Diabetes Care 26:380-386

20. Rosenstock J, Dailey G, Massi-Benedetti M, Fritsche A, Lin Z, Salzman A (2005) Reduced hypoglycemia risk with insulin glargine: a meta-analysis comparing insulin glargine with human NPH insulin in type 2 diabetes. Diabetes Care 28:950 955 